\title{
Research Progress of 5G in the World's Major Countries and Organizations
}

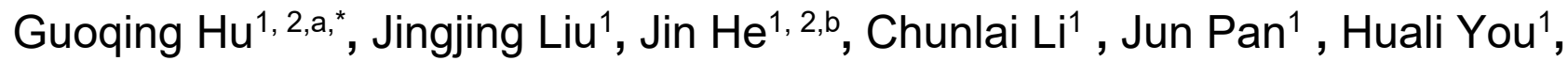

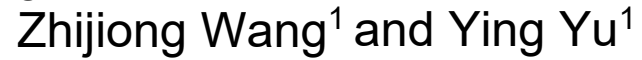 \\ ${ }^{1}$ Peking University Shenzhen SoC Key Laboratory, Peking University Shenzhen Institution, \\ Shenzhen, China \\ ${ }^{2}$ School of Electronics Engineering and Computer Science, Peking University, Beijing, China \\ ahuking@pku.edu.cn, bfrankhe@pku.edu.cn \\ *Corresponding author: huking@pku.edu.cn
}

Keywords: Mobile Communications, 5G, Internet of Things.

\begin{abstract}
In the face of the growth of mass data business brought by mobile Internet and Internet of things, the fifth generation mobile communication system $5 \mathrm{G}$ arise at the historic moment. This paper selects five major 5G driving countries (China, Europe, Japan, South Korea and the United States) and the most important 5G organizations in these countries, and discusses the latest research progress in $5 \mathrm{G}$ area. Through the analysis, we found that $5 \mathrm{G}$ will release the first edition of international standards in 2018 and start a large-scale test network; it is expected that most countries will start the 5G network construction in 2019 and complete the deployment of commercial 5G network in 2020.
\end{abstract}

\section{世界主要国家与组织5G研究进展}

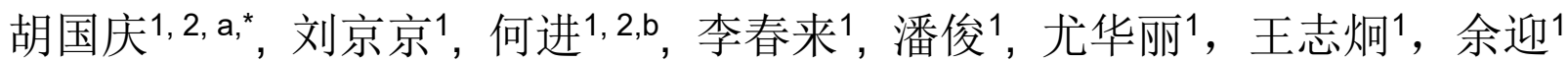

1 北京大学深圳研究院系统芯片设计重点实验室, 深圳, 中国

2北京大学信息科学技术学院, 北京, 中国

ahuking@pku.edu.cn, bfrankhe@pku.edu.cn

*通讯作者: huking@pku.edu.cn

关键词: 移动通信; $5 G$; 物联网。

中文摘要. 面对移动互联网和物联网带来的海量数据业务增长, 第五代移动通信系统 $5 \mathrm{G}$ 应运 而生。本文选取了中欧美日韩五大 $5 \mathrm{G}$ 推动国及这些国家的最重要的 $5 \mathrm{G}$ 推进组织, 探讨了各国 $5 \mathrm{G}$ 领域的最新研究进展。通过分析, 我们发现: 5G将在2018年发布第一版国际标准, 并开始 大规模试验组网; 预计各国将在 2019 年全面启动 $5 \mathrm{G}$ 网络建设, 并在2020年正式完成商用5G网 络的部署。

\section{1. 引言}

移动通信技术自诞生以来, 基本都保持着 “十年一代, 峰值速率十年千倍” 的高速增长。 如图1所示, 与 $1 \mathrm{G}$ 到 $4 \mathrm{G}$ 主要提供面向个人应用不同, $5 \mathrm{G}$ 将渗透到未来社会的各个领域, 全面 拓展到面向产业的应用。根据国际电信联盟ITU公布的相关性能指标, 5G将提供高达几十Gbps 的数据传输速率、千亿量级的接入容量, 可以为增强型移动宽带、超可靠超低时延、万物互 
联等不同场景提供极致业务体验。接下来我们将详细分析世界主要国家和组织在5G方面的主 要贡献与研究进展, 为后续研究提供重要参考。

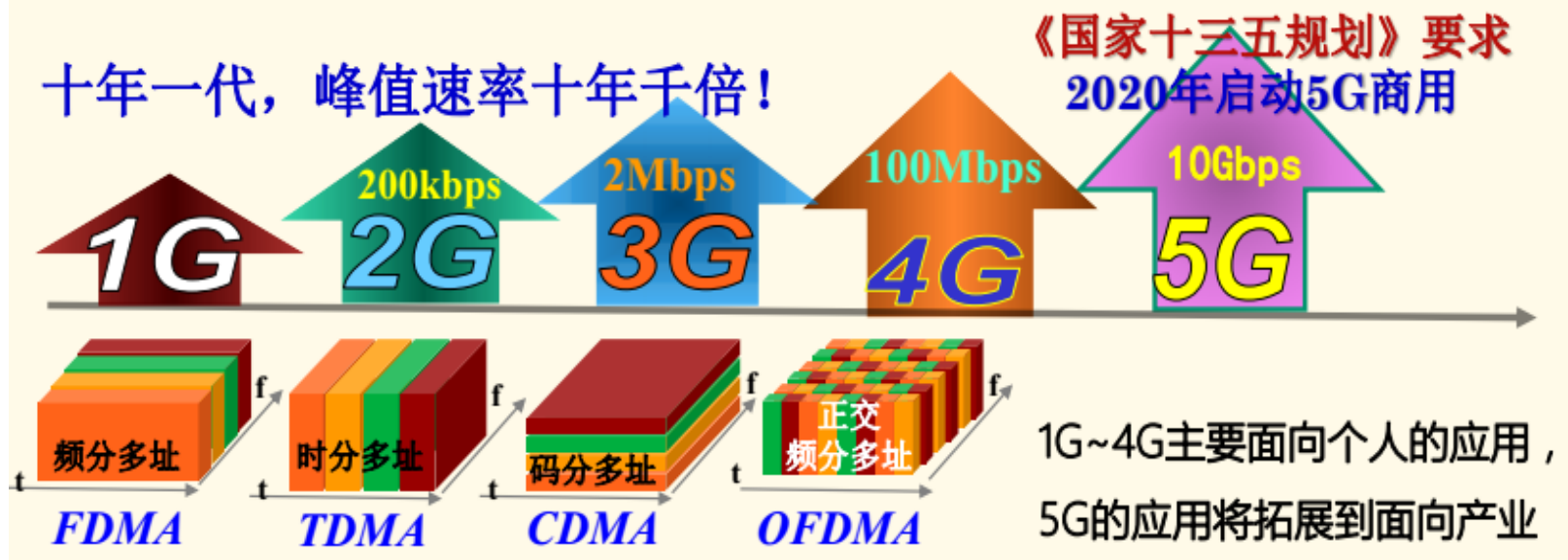

图 1 移动通信技术发展 ${ }^{[1]}$

\section{2. 世界主要国家与组织5G研究进展}

本部分将选取中欧美日韩五大 $5 \mathrm{G}$ 推动国及其组织，探讨 $5 \mathrm{G}$ 的最新研发进展 ${ }^{[2-7]}$ 。

\section{1 欧盟5G研究进展}

早在2012年11月，欧盟就成立了METIS (Mobile and Wireless Communications Enablers for the Twenty-Twenty (2020) Information Society)。2013年12月, 欧盟委员会启动5G公私合营合作 关系(5GPPP)项目, 划拨7亿欧元, 支持 $5 \mathrm{G}$ 关键技术研发。2016年7月, 欧盟发布《欧盟5G宣 言》, 希望使欧洲于 $5 \mathrm{G}$ 网络商业部署方面, 在全球处于领先地位。根据该宣言, 在 2018 年 $3 \mathrm{GPP}$ 发布首批5G国际标准之前, 欧洲各国将以 “独立试验联盟” 的方式开展 $5 \mathrm{G}$ 技术测试, 验证 $5 \mathrm{G}$ 相关技术; 待国际标准发布后, 欧洲通信业界必须就5G技术规范达成一致。2016年11月, 欧 盟委员会无线频谱政策组(RSPG) 公布了相关 $5 \mathrm{G}$ 频谱战略： $700 \mathrm{MHz}$ 将用于 $5 \mathrm{G}$ 广覆盖, 3400-3800MHz是2020年前欧洲5G部署的主要频段; 高频段方面, 24.25-27.5GHz作为毫米波 $5 \mathrm{G}$ 先行频段, $31.8 \mathrm{G}-33.4 \mathrm{GHz}$ 和 40.5G-43.5GHz作为备选频段。2017年12月, 欧盟发布5G路线 图, 详细给出了欧盟在 $5 \mathrm{G}$ 研发领域的主要活动和时间框架, 就 $5 \mathrm{G}$ 频谱的使用和电信运营商的 分配达成一致。2018年3月, 欧盟各国和立法者达成协议, 为5G商业部署开放无线电频谱, 期限为20年。根据该协议，在2020年5G网络将覆盖整个欧盟地区。

\section{2 美国5G研究进展}

美国为推动 $5 \mathrm{G}$ 的发展, 成立了 $5 \mathrm{G}$ Americas组织, 该组织成员已经开展了众多 $5 \mathrm{G}$ 技术测 试工作。2016年7月, 美国联邦通信委员会(FCC)将授权频谱: 27.5G-28.35GHz、37G-38.6GHz、 38.6G-40GHz和免授权频谱：64G-71GHz用于5G部署。2016年10月，美国高通公司发布首款 $5 \mathrm{G}$ 调制解调器骁龙X50: 支持28GHz毫米波频段, 峰值速率高达5Gbps; 2017年10月, 高通展 示了全球第一款 $5 \mathrm{G}$ 手机样机。美国电信运营商方面, 2018年4月AT\&T宣布扩展5G Evolution 技术, 并在2018年底推向全美100多个市场; 2018年5月, 美国电信运营商Verizon宣布将在2018 年底推出 $5 \mathrm{G}$ 网络服务。

\section{3 韩国5G研究进展}

2013-2014年, 韩国启动5GForum并推出了 “5G移动通信促进战略”。2013年5月，韩国三 星首次完成了 $28 \mathrm{GHz}$ 频段 $5 \mathrm{G}$ 测试, 数据传输速率达到 $1 \mathrm{Gbps}$ 。2015年9月, SK电信联合三星 在韩国盆塘部署了一套毫米波 $5 \mathrm{G}$ 通信系统。2018年2月平昌冬奥会上, 三星、韩国电信等公 
司全球率先提供了 $5 \mathrm{G}$ 应用服务，在冬奥会的多个场馆提供了 200 台支持 $5 \mathrm{G}$ 的平板设备，方便 用户使用 $5 \mathrm{G}$ 专用设备查看赛事直播, 提供了包括 360 度VR直播、高清视频传输等在内的十余 种 $5 \mathrm{G}$ 业务。

\section{4 日本5G研究进展}

2014年, 日本成立5GMF(5G移动推进论坛), 以加强产业界、学术界和政府在5G研发领 域的合作; 该组织包括93个单位成员, 下辖战略规划委员会、技术委员会、业务和应用委员 会、网络架构委员会四个研究组, 是日本最重要的5G推动组织。2014年5月, 日本电信营运 商NTT DoCoMo宣布与爱立信、诺基亚、三星等六家厂商共同合作，开始5G相关技术测试。 2016年11月, DoCoMo联合三星完成了高速列车5G测试, 使用28GHz高频频带, 在时速 150 公 里的列车上实现了超过 $2.5 \mathrm{Gbps}$ 的数据传输速率。2017年6月, 在第三届东京湾全球 $5 \mathrm{G}$ 峰会上, NTT DOCOMO联合华为首次完成基于 $3 \mathrm{GPP} 5 \mathrm{G}$ 新空口的 $39 \mathrm{GHz}$ 高频技术测试, 实现了三方实 时4K高清视频会议。2018年1月, 诺基亚宣布与NTT DOCOMO签署5G设备供应协议, 为其提 供 $5 \mathrm{G}$ 通信基站。

\section{5 中国5G研究进展}

2013年，工信部、发改委和科技部等部门共同成立IMT-2020(5G)推进组，对中国5G发展 等重大问题进行总体布局。此外, 中国863计划也分别于2013年6月和2014年3月启动了5G重 大项目一期和二期研发课题。

$\begin{array}{ll}\text { 时间 } & \text { 事件 } \\ 2015 \text { 年 } & \begin{array}{l}\text { 华为、中国移动等企业致力于 } 5 G \text { 技术标准的研发, 中兴通讯大力发展基 } \\ \text { 于 MassiveMimo 的 Pre5G 技术和 } 5 G \text { 标准的研发, 中国 } 5 G \text { 标准有望成 } \\ \text { 为 } 5 G \text { 时代的世界标准。 }\end{array} \\ 2016 \text { 年 1月 } & \text { 正式启动 } 5 G \text { 技术研发试验 }\end{array}$

2016 年 9 月 $5 G$ 第一阶段测试完美收官

\begin{tabular}{|c|c|}
\hline 2016 年 11 月 & IMT-2020(5G)推进组公开发布了《5G 技术研发试验第二阶段技术规范》 \\
\hline 2016 年 11 月 & $\begin{array}{l}\text { 中国移动成功牵头 } 3 G P P \text { 全球统一的 5GS ( } 5 \text { G System Architecture, 5G } \\
\text { 系统架构) 系统设计/标准化 }\end{array}$ \\
\hline 2017 年 & 展开 5G 网络第二阶段测试。 \\
\hline 2018 年 & 进行大规模试验组网。 \\
\hline 2019 年 & 在 2018 年基础上, 启动 $5 \mathrm{G}$ 网络建设。 \\
\hline
\end{tabular}

图 2 中国 $5 \mathrm{G}$ 推动进程 ${ }^{[2]}$

2015年10月, 中国IMT-2020（5G）推进组联合欧盟5G PPP、韩国5G论坛、日本5GMF和 美国5G Americas共同签署了多方合作备忘录 (MoU), 并与2016年5月31日在北京成功举办第 一届全球5G大会。2016年12月, 国务院发布 “十三五” 国家信息化规划, 将 “研发第五代移 动通信 $(5 \mathrm{G})$ 关键核心技术和国际标准以及 $5 \mathrm{G}$ 芯片、终端及系统” 列为十三五期间的重点发展 
项目。如图2所示，2016-2017年，我国5G第一阶段、第二阶段测试业已完成，2018年-2020年 全面部署5G网络。

\section{3. 结束语}

本文选取了中欧美日韩五大 $5 \mathrm{G}$ 推动国及这些国家的最重要的 $5 \mathrm{G}$ 推进组织, 探讨了各国 $5 \mathrm{G}$ 领域的最新研究进展。通过分析, 我们发现: 5G 将在2018年发布第一版国际标准, 并开始 大规模试验组网; 预计各国将在 2019 年全面启动 $5 \mathrm{G}$ 网络建设, 并在2020年正式完成商用5G网 络的部署。

\section{致谢}

本文由深圳市个人创客项目（GRCK2017042415235934），深圳市基础研究项目 （ JCYJ20170817113758285 、 JCYJ20170307164201104 、 JCYJ20160329161334453 、 JCYJ20170817112527562 、 JCYJ20170412153845293 、 JCYJ20170307164247428 、 JCYJ20170817113844300 、 JCYJ20170817112708243 、 JCYJ20170817113029596 、 JCYJ20170817112848591、JCYJ20170412153812353、JCYJ20170307172513653），中国博 士后基金面上资助项目（2017M620523、2017M622619），深圳市技术攻关项目 (JSGG20170414140411874), 国家自然科学基金项目（61574005）, 深港产学研基地产学 研项目（场效应太赫兹器件的关键技术研究）资助。

\section{References}

[1] China Academy of Information and Communications Technology, "The general development trend of 5G", 2015.12.

[2] Yan Zhou, Lin Wang, Jun Yu, Cheng Feng, "China's main push technology becomes the 5G communication code standard", China Merchants Securities Co., Ltd. Industry Research Report, pp.1-20, 2016.11.

[3] Guoqing Hu, Anpeng Huang, Tammy Chang, Xiang Cheng, Hequan Wu, LinzhenXie, Anshi $\mathrm{Xu}$, Zhangyuan Chen, "A Sensor-Based Seamless Handover Solution for Express Train Access Networks (ETANs),” IEEE Communications Letters, vol.16, no.4, pp. 470-472, April 2012.

[4] A. Gupta, R. K. Jha, "A Survey of 5G Network: Architecture and Emerging Technologies", IEEE Access, vol.3, pp.1206-1232, 2015.08.

[5] T. S. Rappaport, Y. Xing, G. R. MacCartney, Jr., A. F. Molisch, E. Mellios, and J. Zhang, "Overview of millimeter wave communications for fifth-generation $(5 \mathrm{G})$ wireless networks," IEEE Transactions on Antennas and Propagation, vol.65, no.12, pp.6213-3230, Dec. 2017.

[6] Guoqing Hu, Anpeng Huang, Ruisi He, Bo Ai, Zhangyuan Chen, "Theory analysis of the handover challenge in Express Train Access Networks (ETAN)", China Communications, vol. 11, no.7, pp. 92-98, July 2014.

[7] J. Huang, C.-X. Wang*, R. Feng, J. Sun, W. Zhang, and Y. Yang, "Multi-frequency MmWave massive MIMO channel measurements and characterization for $5 \mathrm{G}$ wireless communication systems,” IEEE J. Sel. Areas Commun., vol. 35, no. 7, pp. 1591-1605, July 2017. 